\title{
Total Intercarrier Interference Cancellation for OFDM Mobile Communication Systems
}

\author{
Xue $\mathrm{Li}^{1}$, Ruolin Zhou ${ }^{1}$, Vasu Chakravarthy ${ }^{2}$, Steven Hong ${ }^{3}$, and Zhiqiang $\mathrm{Wu}^{1}$ \\ Wright State University ${ }^{1}$, Air Force Research Laboratory ${ }^{2}$, Stanford University ${ }^{3}$
}

\begin{abstract}
For orthogonal frequency division multiplexing (OFDM) communication systems, the orthogonality among subcarriers is lost in mobile radio channels due to the frequency offsets caused by mobility. As a direct result, intercarrier interference (ICI) is observed on each and every subcarrier, leading to significant performance degradation. Many ICI cancellation methods such as windowing and frequency domain coding have been proposed in the literature to cancel ICI and improve the BER performance of OFDM in mobile channel. However, the performance improvement achieved by all the existing ICI cancellation methods is far from enough: the BER performance after ICI cancellation is still much worse than the BER performance of original OFDM without ICI. Moreover, popular ICI cancellation methods like ICI self-cancellation reduce ICI at the price of lowering the transmission rate and reducing the bandwidth efficiency. Other frequency-domain coding methods do not reduce the data rate, but produce less reduction in ICI as well. In this paper, we propose a novel ICI cancellation scheme which can eliminate the ICI entirely and offer a OFDM mobile system with the same BER performance of a OFDM system without ICI. More importantly, the proposed ICI cancellation scheme, namely Total ICI Cancellation, does not lower the transmission rate or reduce the bandwidth efficiency. Specifically, the Total ICI Cancellation scheme takes advantage of the orthogonality of the ICI matrix and offers perfect ICI cancellation and significant BER improvement at linearly growing cost. Simulation results in AWGN channel and multi-path fading channel confirm the superb performance of the proposed Total ICI Cancellation scheme in the presence of frequency offset or time variations in the channel, outperforming all the existing ICI cancellation methods.
\end{abstract}

\section{INTRODUCTION}

Orthogonal Frequency Division Multiplexing (OFDM) has been considered a strong candidate for next generation high-data-rate wireless communication systems [1]. However, OFDM is not suitable for mobile communication systems due to the frequency offset introduced by Doppler shift in high mobility environment. With this frequency offset, the orthogonality among all the OFDM subcarriers is lost and intercarrier interference (ICI) is generated.

Many methods have been proposed in the literature to mitigate the frequency-offset problem to cancel the ICI for OFDM system. Most of such methods use signal processing and/or coding to reduce the sensitivity of the OFDM system to the frequency offset. For example, in [2], authors developed low-complexity minimum mean-square error and decision-feedback equalizer receivers to suppress ICI based on the fact that the ICI power mainly comes from a few neighboring subcarriers. Some researchers also proposed a lot of schemes to estimate the frequency offset, including data aided estimation [3][4] and blind estimation [5][6][7]. In the light of the same statement, an effective methods known as the ICI self-cancellation scheme has been proposed in [8] where copies of the same data symbol are modulated on $L$ adjacent subcarriers using optimized weights. In [9], a generalized ICI self-cancellation scheme has been proposed. In [10], a ICI self-cancellation using data-conjugate method is proposed.

However, all existing ICI cancellation methods are not without their drawbacks. Even though all the existing ICI cancellation methods reduce ICI and improve BER performance for OFDM system, the performance improvement is very limited. The BER performance after ICI cancellation is still significantly worse than the original OFDM system without ICI. More important, most of the existing ICI cancellation methods achieve the ICI reduction and BER performance improvement at the cost of lowering the transmission rate and reducing the bandwidth efficiency. There do exist some methods that do not reduce the date rate, however, such methods produce even less reduction in ICI.

It has been observed that the ICI matrix in OFDM is an orthogonal matrix. Hence, from the receiver side, a OFDM with ICI can be considered as a MC-CDMA (Multi-carrier CDMA) system where all the $N$ data symbols carried by the OFDM transmission are spread over all $N$ subcarriers. However, since the frequency offset is time varying and unknown at the receiver side, the spreading code matrix of the equivalent MC-CDMA system is unknown. Hence, it has been proposed to transmit training sequences to estimate the frequency offset and cancel ICI via the estimated frequency offset. Of course, by doing so, some data rate needs to be allocated for the training sequence and complicated frequency offset estimation algorithms need to be implemented at receiver side.

In this paper, we propose a brand new approach to solve the ICI problem in mobile OFDM system without estimating frequency offset through training symbols (and without data rate reduction). Specifically, we propose to quantize the normalized frequency offset into $M$ discrete values, leading to $M$ spreading code matrices as candidates. Next, by decoding the received signal using these $M$ spreading code matrices, $M$ decisions are made on the data symbols. Using these $M$ data symbols to recreate the received signal with ICI and measuring the Euclidean distance of the $M$ recreated signals with the actual received signal, the best normalized frequency offset is chosen and the best corresponding data symbols are determined. Simulation results over AWGN channel and mobile multi-path fading channel demonstrate that not only 
the proposed system effectively eliminates ICI entirely and offer the best BER performance available which matches the BER performance of OFDM system without ICI, it achieves the superb performance with reasonable computational complexity. It is shown that the complexity of the proposed system is linearly growing with the number of quantization levels $M$, and $M$ does not have to be a big number to achieve the best performance. Simulation results show that we only need to pick $M=5$.

The rest of the paper is organized as follows: Part II reexamine the ICI of mobile OFDM systems and prove that the received OFDM signal with the presence of ICI can be considered as an orthogonal MC-CDMA system. Part III describes the proposed Total ICI Cancellation method. Part IV provides simulation results over AWGN channel and multipath fading channels. Conclusion follows.

\section{ICI OF OFDM SYSTEMS AND ORTHOGONALITY}

It is well known that the received OFDM signal on subcarrier $k$ in AWGN channel with ICI is

$$
\begin{array}{r}
Y(k)=X(k) S(0)+\sum_{l=0, l \neq k}^{N-1} X(l) S(l-k)+n_{k}, \\
k=0,1, \ldots, N-1
\end{array}
$$

where $N$ is the total number of the subcarriers, $X(k)$ denotes the transmitted symbol $(X(k) \in\{+1,-1\}$ if BPSK is employed, for example) for the $k^{t h}$ subcarrier, $n_{k}$ is the additive Gaussian noise sample. The sequence $S(l-k)$ is the ICI coefficient between $l^{t h}$ subcarrier and $k^{t h}$ subcarrier:

$$
\begin{aligned}
S(l-k)= & \frac{\sin (\pi(\varepsilon+l-k))}{N \sin \left(\frac{\pi}{N}(\varepsilon+l-k)\right)} \\
& \cdot \exp \left(j \pi\left(1-\frac{1}{N}\right)(\varepsilon+l-k)\right)
\end{aligned}
$$

where $\varepsilon$ is the normalized frequency offset given by $\varepsilon=\frac{\Delta F}{\Delta f}$, $\Delta F$ is the frequency offset, $\Delta f$ is the subcarrier bandwidth of the OFDM system. It is reasonable to assume that $0 \leq \varepsilon<1$.

Now, denote vector $\vec{X}$ as the transmitted symbol $\vec{X}=$ $\{X(0), X(1), \ldots, X(N-1)\}$, vector $\vec{Y}$ as the received signal vector $\vec{Y}=\{Y(0), Y(1), \ldots, Y(N-1)\}$, and $\vec{n}=$ $\left\{n_{0}, n_{1}, \ldots, n_{N-1}\right\}$, we have:

$$
\vec{Y}=\vec{X} \mathbf{S}+\vec{n}
$$

where $\mathbf{S}$ is the ICI coefficient matrix, and the $p^{t h}$ row and $q^{t h}$ column element of $N \mathrm{x} N$ matrix $\mathbf{S}$ is

$$
\mathbf{S}_{p, q}=S(p-q)
$$

and the matrix $\mathbf{S}$ corresponds to

$$
\mathbf{S}=\left[\begin{array}{cccc}
S(0) & S(-1) & \ldots & S(1-N) \\
S(1) & S(0) & \ldots & S(2-N) \\
\vdots & \vdots & \ddots & \vdots \\
S(N-1) & S(N-2) & \ldots & S(0)
\end{array}\right]
$$

From equation (3), it is obvious that the received signal can be viewed as a MC-CDMA (multi-carrier code division multiple access) signal with $N$ users, the $k^{t h}$ user's information symbol is $X(k)$, and the $k^{t h}$ user's spreading code is the $k^{t h}$ column of matrix $\mathbf{S}$.

Now, it is important to note that the ICI coefficient matrix $\mathbf{S}$ is an orthogonal matrix, i.e.,

$$
\mathbf{S S}^{\prime}=\mathbf{I}
$$

where $\mathbf{S}^{*}$ is the conjugate transpose of matrix $\mathbf{S}$ and $\mathbf{I}$ is identity matrix.

Hence, the OFDM signal with ICI at receiver side can be considered as an orthogonal MC-CDMA system with spreading code matrix $\mathbf{S}$. As a direct result, the ICI can be totally removed from the OFDM signal if we apply a matrix multiplication to the received signal vector $\vec{Y}$ :

$$
\vec{R}=\vec{Y} \mathbf{S}^{\prime}=\vec{X}+\vec{n} \mathbf{S}^{\prime}
$$

Next, we can simply make decision of $\vec{X}$ based on the sign of $\vec{R}$. Since $\mathbf{S}^{\prime}$ is also an orthogonal matrix, the noise vector $\vec{n} \mathbf{S}^{\prime}$ in $\vec{R}$ has the same covariance matrix as that of $\vec{n}$. Hence, the entire ICI is eliminated and the BER performance would be the same of a OFDM system without ICI at all.

Of course, the problem is: the receiver does not know the spreading code matrix $\mathbf{S}$ because the normalized frequency offset $\varepsilon$ is unknown. Hence, it has been proposed to estimate the normalized frequency offset $\varepsilon$ through some training symbols. Of course, by doing so, some data rate needs to be allocated for the training symbols, and sophisticated frequency offset estimation algorithms need to be implemented at receiver side.

\section{TOTAL ICI CANCELLATION FOR OFDM}

Here, we propose the Total ICI Cancellation scheme to eliminate ICI on mobile OFDM systems without transmitting any training symbols (and reducing data rate). While the normalized frequency offset $\varepsilon$ is unknown to the receiver, we can quantize $\varepsilon$ into $M$ equally spaced values:

$$
\varepsilon_{m}^{\prime}=m \cdot \Delta \varepsilon, m=0,1, \ldots, M-1
$$

where $\Delta \varepsilon$ is the quantization level of normalized frequency offset, and $M$ is the number of quantization levels:

$$
\Delta \varepsilon=\frac{1}{M}, m=0,1, \ldots, M-1
$$

One of these $M$ quantized $\varepsilon^{\prime}$ s is closet to the true $\varepsilon$.

Now, let's build $M$ parallel branches at the receiver. Each branch uses one of the $M$ quantized $\varepsilon^{\prime}$ s to create the corresponding ICI coefficient matrix $\tilde{\mathbf{S}}$. Hence, we have $M$ ICI 
coefficient matrices $\tilde{\mathbf{S}}_{0}, \tilde{\mathbf{S}}_{1}, \ldots, \tilde{\mathbf{S}}_{N-1}$ where the $m^{\text {th }}$ matrix corresponds to:

$$
\tilde{\mathbf{S}}_{\mathbf{m}}=\left[\begin{array}{cccc}
S_{m}(0) & S_{m}(-1) & \ldots & S_{m}(1-N) \\
S_{m}(1) & S_{m}(0) & \ldots & S_{m}(2-N) \\
\vdots & \vdots & \ddots & \vdots \\
S_{m}(N-1) & S_{m}(N-2) & \ldots & S_{m}(0)
\end{array}\right]
$$

and

$$
\begin{aligned}
S_{m}(l-k)= & \frac{\sin \left(\pi\left(\varepsilon_{m}^{\prime}+l-k\right)\right)}{N \sin \left(\frac{\pi}{N}\left(\varepsilon_{m}^{\prime}+l-k\right)\right)} \\
& \cdot \exp \left(j \pi\left(1-\frac{1}{N}\right)\left(\varepsilon_{m}^{\prime}+l-k\right)\right)
\end{aligned}
$$

Using these $M$ matrices, we can have $M$ decisions on the transmitted data vector $\vec{X}$ where the $m^{\text {th }}$ branch will make decision on the estimation of $\vec{X}$ as:

$$
\hat{\vec{X}}_{m}=\operatorname{sgn}\left\{\vec{Y} \tilde{\mathbf{S}}_{\mathbf{m}}^{\prime *}\right\}
$$

where $\operatorname{sgn}(X)$ presents the sign of $X$.

Next, with the data vector estimation $\hat{\vec{X}}_{m}$, each branch can reproduce the received signal $\overrightarrow{\vec{Y}}_{m}$ by using the data vector estimation $\hat{\vec{X}}_{m}$, the ICI coefficient matrix of that branch $\tilde{\mathbf{S}}_{\mathbf{m}}$ :

$$
\hat{\vec{Y}}_{m}=\hat{\vec{X}}_{m} \tilde{\mathbf{S}}_{\mathbf{m}}
$$

It is easy to understand that the one branch whose $\varepsilon_{m}^{\prime}$ is closest to the true value of $\varepsilon$ should reproduce the received signal $\hat{\vec{Y}}_{m}$ also closest to the received signal vector $\vec{Y}$. Hence, we only need to calculate and compare the Euclidean distances between the $M$ reproduced received signal vectors $\hat{\vec{Y}}_{m}$ and the truly received signal vector $\vec{Y}$ and pick the one with the minimum distance to be the best branch and use that branch's estimated data vector as the final decision:

$$
\hat{\vec{X}}=\operatorname{argmin}\left\{\left\|\hat{\vec{Y}}_{m}-\vec{Y}\right\|^{2}\right\}
$$

where $\left\|\hat{\vec{Y}}_{m}-\vec{Y}\right\|^{2}$ represents the Euclidean distance between vector $\overrightarrow{\vec{Y}}_{m}$ and vector $\vec{Y}$.

It is important to note that the complexity of the proposed Total ICI Cancellation method is linearly growing with the quantization level $M$, keeping the computational complexity at reasonable range. For each OFDM symbol, the Total ICI Cancellation scheme requires $2 M$ matrix multiplications and $M$ comparisons. The increased complexity is not significant, especially when $M$ is small. As we will show in the next Section, we don't need to use a huge $M$ to achieve the best performance. In all the cases, $M=8$ is good enough to provide perfect ICI cancellation and superb BER performance matching the lower bound.

The block diagram of the proposed Total ICI Cancellation scheme is shown in Figure 1.

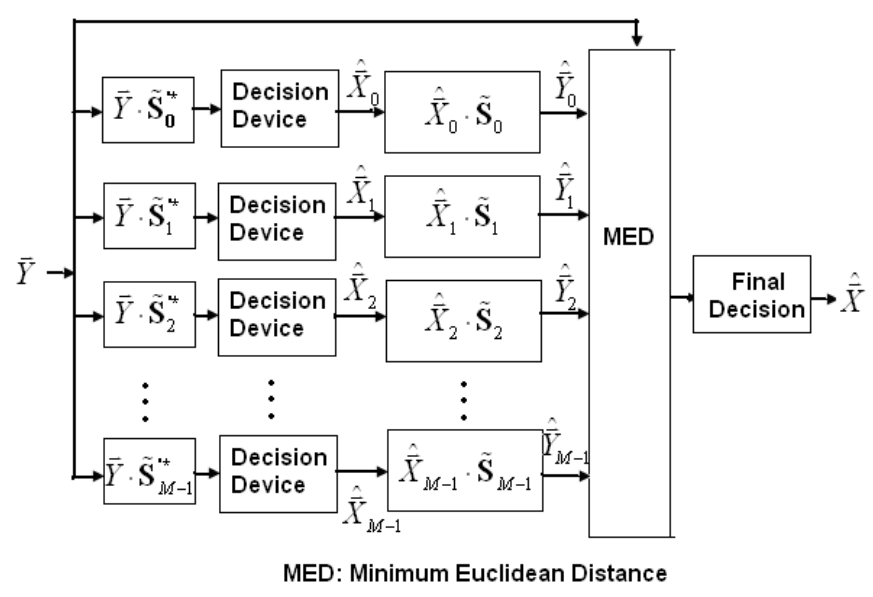

Fig. 1. Block Diagram of the Total ICI Cancellation

\section{AnAlysis in MUltipath FADING Channels}

In a multipath fading channel, let's denote the complex fading gain on the $k^{t h}$ subcarrier is $\alpha_{k}$. Then the received OFDM signal after transmission through such a fading channel with frequency offset is:

$$
\vec{Y}=\vec{X} \alpha \mathbf{S}+\vec{n}
$$

where $\alpha$ is a diagonal matrix $\alpha=\operatorname{diag}\left\{\alpha_{0}, \alpha_{1}, \ldots, \alpha_{N-1}\right\}$.

Similar to the analysis in AWGN channel, the received OFDM signal represented in equation (15) can also be viewed as a $N$ user MC-CDMA system with spreading code matrix $\mathbf{S}$ and the $k^{t h}$ user's data symbol is $\alpha_{k} X(k)$. Hence, if the spreading code matrix $\mathbf{S}$ is known, we can eliminate the ICI by multiplying $\mathbf{S}^{\prime *}$ to the received vector $\vec{Y}$.

So the Total ICI Cancellation schemes works the same way as in AWGN channel with only one exception: the fading channel characteristics $\alpha$ needs to be estimated at the receiver side (which is required for OFDM transmission) and the reproduced received signal vector now has to consider the fading effects:

$$
\hat{\vec{Y}}_{m}=\hat{\vec{X}}_{m} \alpha \tilde{\mathbf{S}}_{\mathbf{m}}
$$

\section{Simulation Results}

In this section, we use numerical simulation results to present the effectiveness of the proposed Total ICI Cancellation scheme. We provide BER simulation results for the proposed Total ICI Cancellation scheme in both AWGN channel and multi-path fading channels. All the systems are assumed to have $N=32$ subcarriers and employ BPSK modulation.

\section{A. AWGN Channel with a Constant Frequency Offset}

The simplest way to examine the effectiveness of the proposed Total ICI Cancellation scheme is to transmit signals through a AWGN channel with a constant frequency offset between the transmitter and receiver. Figure 2 illustrates the simulation result when the normalized frequency offset (NFO) $\varepsilon=0.1$ and Figure 3 shows the case when $\varepsilon=0.3$. In the Total 
ICI Cancellation scheme, we use $M=10$. In both of the two figures, the blue line shows the BER performance of OFDM without ICI, the green line marked with circles represents the performance of OFDM with ICI, and the red line marked with stars represents that of our proposed Total ICI Cancellation scheme. Since we chose $M=10$, so the quantization level of normalized frequency offset $\Delta \varepsilon=0.1$. Hence, one of the $M$ branches actually has the perfect ICI coefficient matrix to work with. To prove the effectiveness of our Total ICI Cancellation method in all scenarios, Figure 4 presents the results when $\varepsilon=0.287532$ so none of the $M$ branches matches the actual $\varepsilon$. It is obvious from these figures that when the normalized frequency offset $\varepsilon$ increases, the BER performance of OFDM significantly degrades, but the Total ICI Cancellation scheme eliminates the ICI and provides the same BER performance as that of a OFDM without ICI.

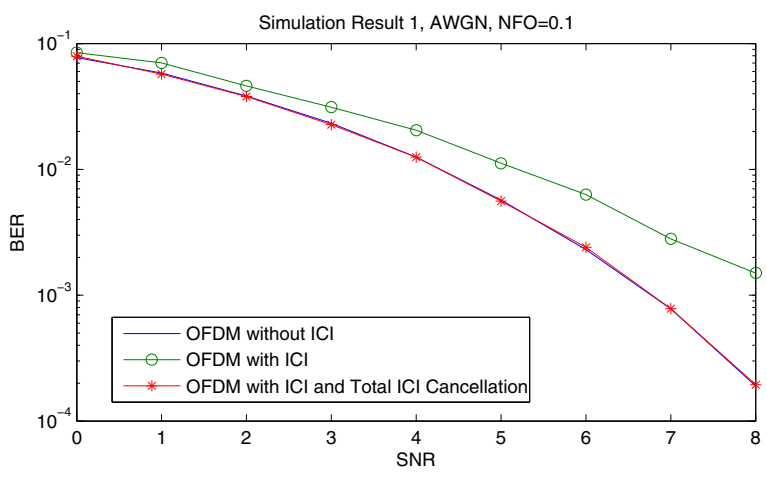

Fig. 2. Simulation 1 in $\mathrm{AWGN}$ and $\mathrm{NFO}=0.1$

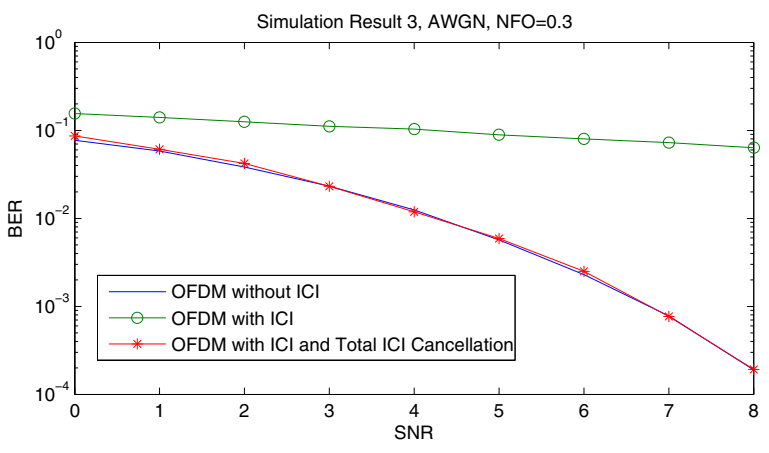

Fig. 3. Simulation 3 in $A W G N$ and $\mathrm{NFO}=0.3$

Figure 5 shows the BER performance versus normalized frequency offset $\varepsilon$. It is evident that with the increase of $\varepsilon$, the BER performance of OFDM significantly degrades while the OFDM with Total ICI Cancellation keeps the same performance despite the frequency offset.

\section{B. Multipath Mobile Channels}

In a practical mobile multipath radio channel, time-variant multipath propagation leads to Doppler frequency shift which is a random variable. Here we measure the performance of the proposed Total ICI Cancellation method in multipath fading

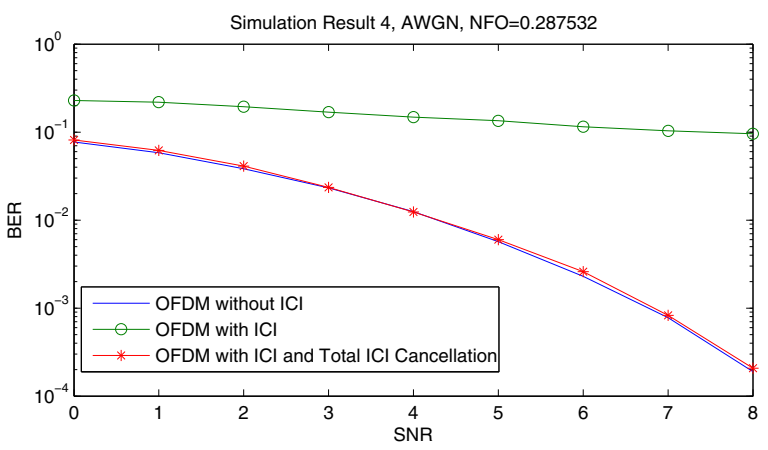

Fig. 4. Simulation 4 in $\mathrm{AWGN}$ and $\mathrm{NFO}=0.287532$

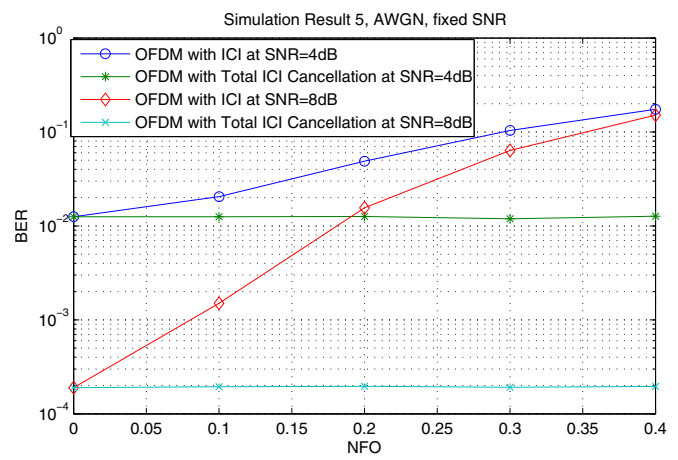

Fig. 5. Simulation 5 in AWGN at Fixed SNR

channels. As a measure of Doppler frequencies, we use the normalized maximum Doppler spread $\varepsilon_{B}$, which is defined as the ratio between the channel maximum Doppler spread to the subcarrier bandwidth. We use the Hilly Terrain (HT) channel models defined by the GSM standard as our channel model. Total number of subcarriers is also assumed to be 32 .

Figure 6 shows the case when $\varepsilon_{B}=0.2$ and Figure 7 shows the case when $\varepsilon_{B}=0.3$. In the Total ICI Cancellation scheme, we use $M=10$. In both of the two figures, the blue line shows the BER performance of OFDM without ICI, the green line marked with circles represents the performance of OFDM with ICI, and the red line marked with stars represents that of our proposed Total ICI Cancellation scheme. It is evident from these figures that the proposed Total ICI Cancellation entirely eliminates the effect of ICI and matches the performance of the OFDM without ICI in fading channels as well.

Figure 8 illustrates the effect of the number of normalized frequency offset quantization levels $M$ on the performance of the proposed Total ICI Cancellation scheme. In Figure 8, three BER versus $M$ curves of different SNRs are shown. It is easy to understand that when $M$ increases, more quantization levels are used and better ICI coefficient matrix estimation is achieved, so the performance of the proposed scheme will also improve. As shown in Figure 8, when $M$ is very small, the proposed Total ICI Cancellation scheme actually offers pretty bad performance due to the large quantization error. However, when $M$ increases, the Total ICI Cancellation converges fast and provide ICI cancellation and BER improvement quickly. 


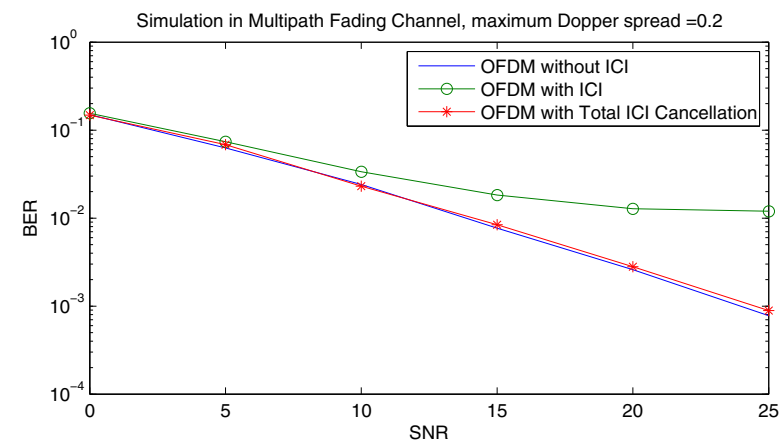

Fig. 6. Simulation 2 in Fading Channel and normalized maximum Doppler spread $=0.2$

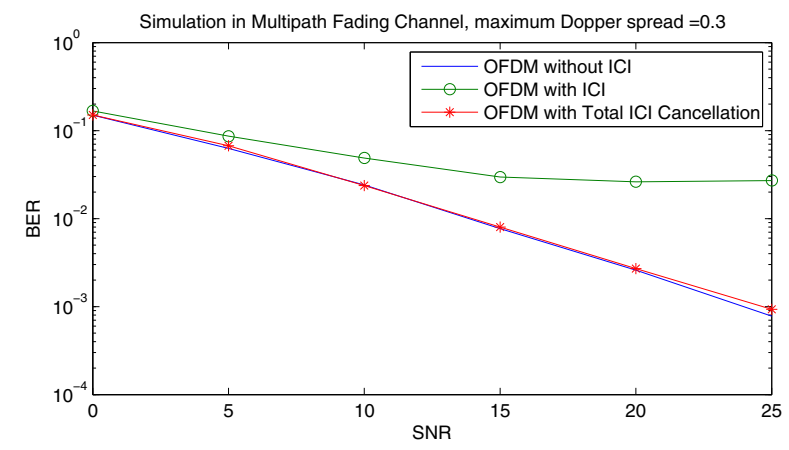

Fig. 7. Simulation 3 in Fading Channel and normalized maximum Doppler spread $=0.3$

When $M$ is larger than 5 , there is no noticeable performance gain to increase the quantization level. This can be explained as the following: when the quantization step $\Delta \varepsilon$ is small enough, the Total ICI Cancellation's ICI cancellation capability is enough to remove all the intercarrier interference and there is no need to decrease $\Delta \varepsilon$ anymore. It is evident from Figure 8 that the computational complexity of the proposed scheme is very reasonable.

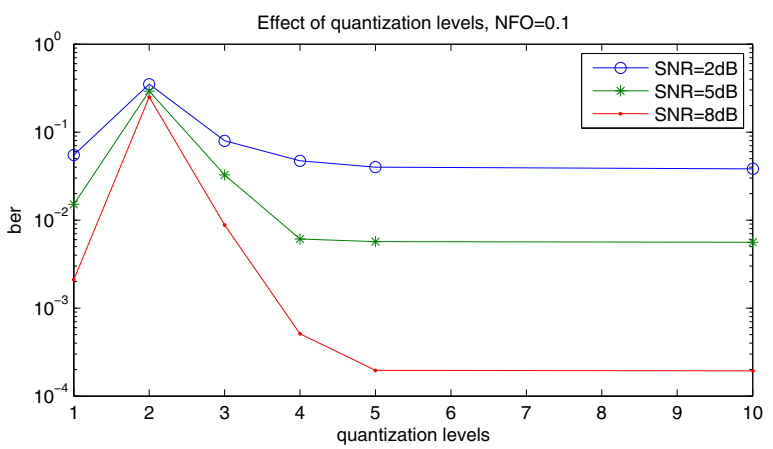

Fig. 8. Effect of NFO Quantization Levels, AWGN, NFO=0.1

\section{CONCLUSION}

In this paper, we proposed a novel intercarrier interference cancellation scheme called Total ICI Cancellation for mobile OFDM systems. Taking advantage of the orthogonality of the ICI coefficient matrix, the proposed ICI cancellation scheme can eliminate the ICI experienced in mobile OFDM systems entirely and provide significant BER improvement which matches the BER performance of OFDM system without ICI at all. The proposed Total ICI Cancellation scheme not only provides perfect performance, it doesn't reduce the bandwidth efficiency of OFDM system like many existing ICI cancellation methods do. Simulations over AWGN channel and multipath fading channel confirm the effectiveness of the proposed scheme. Finally, the Total ICI Cancellation scheme achieves such superb performance at a very reasonable computational complexity which linearly grows with the number of normalized frequency offset quantization.

\section{ACKNOWLEDGEMENT}

This material is based upon work supported by the National Science Foundation under Grant No. 0708469, No. 0737297, No. 0837677, the Wright Center for Sensor System Engineering, and the Air Force Research Laboratory. Any opinions, findings, and conclusions or recommendations expressed in this material are those of the author(s) and do not necessarily reflect the views of the funding agencies.

\section{REFERENCES}

[1] W. Y. Zou and Y. Wu, "Cofdm: an overview," IEEE Transactions on Broadcasting, vol. 41, pp. 1-8, March 1995.

[2] X. Cai and G. B. Giannakis, "Bounding performance and suppressing intercarrier interference in wireless mobile ofdm," IEEE Transactions on Communications, vol. 51, pp. 2047-2056, December 2003.

[3] H. Minn, P. Tarasak, and V. Bhargava, "Ofdm frequency offset estimation based on blue principle," IEEE Vehicular Technology Conference, vol. 2, pp. $1230-1234$, September 2002.

[4] A. J. Coulson, "Maximum likelihood synchronization for ofdm using a pilot symbol: analysis," IEEE Journal on Selected Areas in Communications, vol. 19, no. 12, pp. 2495 - 2503, 2001.

[5] Y. Yao and G. B. Giannakis, "Blind carrier frequency offset estimation in siso, mimo, and multiuser ofdm systems," IEEE Transactions on Communications, vol. 53, pp. 173-183, Jan. 2005.

[6] L. Wu, X.-D. Zhang, P.-S. Li, and Y.-T. Su, "A blind cfo estimator based on smoothing power spectrum for ofdm systems," IEEE Transactions on Communications, vol. 57, pp. 1924-1927, July 2009.

[7] F. Yang, K. H. Li, and K. C. Teh, "A carrier frequency offset estimator with minimum output variance for ofdm systems," IEEE Communications Letters, vol. 8, pp. 677-679, Nov. 2004.

[8] Y. Zhao and S.-G. Haggman, "Intercarrier interference self-cancellation scheme for ofdm mobile communication systems," IEEE Transactions on Communications, vol. 49, pp. 1185-1191, July 2001.

[9] A. Seyedi and G. J. Saulnier, "General ici self-cancellation scheme for ofdm systems," IEEE Transactions on Vehicular Technology, vol. 54, pp. 198-210, Janurary 2005.

[10] H.-G. Ryu, Y. Li, and J.-S. Park, "An improved ici reduction method in ofdm communication system," IEEE Transactions on Boradcasting, vol. 51, pp. 395-400, September 2005. 